\title{
Dynamical trajectory of glucocorticoids tapering and discontinuation in patients with rheumatoid arthritis commencing glucocorticoids with csDMARDs: a real- world data from 2009 to 2020
}

\author{
Wenhui Xie (1) , Hong Huang (1) Guangtao Li, Yanjie Hao, Yanni Gui, Yu Wang, \\ Xuerong Deng, Juan Zhao ํ) , Yan Geng, LanLan Ji, Xiaohui Zhang, Zhibo Song, \\ Zhuoli Zhang
}

\begin{abstract}
Handling editor Josef $S$
Smolen

- Additional material is published online only. To view, please visit the journal online (http://dx.doi.org/10.1136/ annrheumdis-2021-220112).

Department of Rheumatology and Clinical Immunology, Peking University First Hospital, Beijing, China
\end{abstract}

Correspondence to Professor Zhuoli Zhang Rheumatology and Clinical Immnunology, Peking University First Hospital, Beijing, Beijing, China; zhuoli.zhang@126.com

Received 7 February 2021 Revised 21 March 2021 Accepted 23 March 2021 Published Online First 2 April 2021

Check for updates

(C) Author(s) (or their employer(s)) 2021. No commercial re-use. See rights and permissions. Published by BMJ.

To cite: Xie $\mathrm{W}$, Huang $\mathrm{H}$ Li G, et al. Ann Rheum Dis 2021;80:997-1003

\section{ABSTRACT}

Objective To unravel the dynamical trajectory and features of glucocorticoids (GC) tapering and discontinuation in patients with rheumatoid arthritis (RA) commencing GC with concomitant conventional synthetic disease-modifying antirheumatic drugs (csDMARDs).

Methods We used data from longitudinal real-world Treat-to-TARget in RA cohort. Patients with RA who started GC and contaminant csDMARDs therapy were included. The changes in GC dose and disease activity were evaluated. GC discontinuation rate was analysed using Kaplan-Meier analysis. The relapse profile within 6 months after GC discontinuation was also analysed. Results A total of 207 patients with RA were included. During a median follow-up of 38.6 months, 124 patients discontinued GC. The median prednisolone dose of $10(5-10) \mathrm{mg} /$ day at initiation was reduced by $50 \%$ in the first 6 months and then more slowly, to zero by 48 months eventually. The cumulative probabilities of GC discontinuation were $9.7 \%, 26.6 \%, 48.0 \%$ and $58.6 \%$ at month 6 , years 1, 2 and 3 , with calculated median time to $\mathrm{GC}$ cessation of 27 months. In 110 DMARD-naïve patients, the corresponding cumulative probabilities of GC discontinuation were, respectively, $12.7 \%, 30.0 \%$, $50.9 \%$ and $60.6 \%$, with calculated median time to $\mathrm{GC}$ cessation of 24 months. Of the 124 patients who discontinued GC, adding other csDMARDs or concomitant csDMARDs increment was documented in $28.2 \%$ of them. Approximately half of 124 patients were in clinical remission at GC discontinuation. Within 6 months after GC withdrawal, $79.1 \%(91 / 115)$ of patients maintained relapse free.

Conclusions In patients with RA commencing $\mathrm{GC}$ besides csDMARDs, GC is feasibly discontinued with favourable control of disease activity in reallife setting, mostly without short-term flare. But the withdrawal time is far from reaching the recommended time frame, indicating the gap between real-world practice and current guidelines.

\section{INTRODUCTION}

Glucocorticoids (GC) are effective in treating rheumatoid arthritis (RA), and currently still plays a crucial role in the management of RA. Its

\section{Key messages}

What is already known about this subject?

- Glucocorticoids (GC) are currently recommended as bridging therapy in combination with conventional synthetic disease-modifying antirheumatic drugs (csDMARDs) in patients with rheumatoid arthritis (RA), but should be tapered as rapidly as clinically feasible for safety concerns about its long-term use.

- The clinical dynamical trajectory and characteristics of GC cessation when initiating GC with csDMARDs therapy remains completely unknown, especially in real-life setting.

What does this study add?

- In patients with RA initiating GC and concomitant csDMARDs, the median prednisolone dose was reduced by $50 \%$ in the first 6 months, and then more slowly, to zero by 48 months eventually. Cumulative probabilities of GC discontinuation were $26.6 \%, 48.0 \%$ and $58.6 \%$ at year 1 , year 2 , and year 3 , with calculated median time to GC cessation of 27 months.

- Approximately half of patients achieved clinical remission at GC discontinuation, and more than two-thirds of them maintained flare free within 6 months after GC cessation.

How might this impact on clinical practice or future developments?

- In general, tapering/stopping GC in patients with RA with concomitant csDMARDs is feasible, when disease activity is favourable. But the withdrawal time is far from reaching the recommended time frame, indicating the gap between clinical practice and current guidelines.

- Adding a targeted DMARDs seems to be required in most patients to facilitate $\mathrm{GC}$ discontinuation within the time frame of about 3 months in accordance with current guidelines.

disease-modifying potentials to improve clinical, functional and structural outcomes when combined with conventional synthetic disease-modifying anti-rheumatic drugs (csDMARDs) have been well 
documented in controlled studies. ${ }^{1-5}$ GC can not only significantly reduce the inflammation, but also rapidly relieve the articular symptoms in contrast to csDMARDs. ${ }^{4-6}$ In the recommendations by the European League Against Rheumatism (EULAR) for the management of RA, short-term GC should be considered as a therapy bridging csDMARDs to exhibit efficacy. ${ }^{2}{ }^{7}$ Similarly, short-term GC was recommended in 2015 American College of Rheumatology (ACR) guidelines for both patients with early and established RA with moderate or high disease activity despite of using csDMARDs or biological DMARDs (bDMARDs). ${ }^{8}$

In general, GC use in RA is still strongly debated, mostly focusing on the length and dose. In recent years, the safety concerns of its long-term use, even at low dose, have been increasing, such as infection, diabetes mellitus, atherosclerosis and osteoporosis. ${ }^{9-13}$ In daily practice, patients with RA and rheumatologists also concerned of the safety of GC therapy. ${ }^{6}$ Currently, the 2019 EULAR guidelines underline that GC should be tapered as rapidly as clinically feasible, and ultimately stopped, ideally within 3 months. ${ }^{2}$ Nevertheless, there is little evidence to guide clinicians to taper GC, leading to widely variable practice patterns. ${ }^{14}{ }^{15}$ Currently, long-term use of GC is common, although GC tapering and discontinuation are explicitly supported by RA management guidelines. For example, in a French prospective cohort of 397 patients who received GC, $55 \%$ were exposed to GC for over 2 years and $11.8 \%$ patients for up to 9 years. ${ }^{10}$ To date, the clinical trajectory and characteristics of GC cessation in patients with RA with GC plus csDMARDs therapy remains completely unknown, especially in real-life setting. To fill the gap, we performed the present study to unravel the profiles of dynamic changes of GC dose and feasibility of GC withdrawal in the context of combination with csDMARDs in real-world practice.

\section{MATERIALS AND METHODS}

\section{Study design and participants}

The Treat-to-TARget in RA (TARRA) cohort is a longitudinal observational cohort that includes patients with RA of Peking University First Hospital. The cohort, first established over a decade ago, has been described in greater detail in our previous researches. ${ }^{16-21}$ Briefly, demographics, symptom duration, RA core set variables, laboratory findings and treatment details were documented at the initial visit in the database. After that, all patients are prospectively followed up and documented at least 3 monthly for those in moderate/high diseases activity and every 3-12 months (usually 3-6 months) for those in remission/low disease activity (LDA). Additional follow-up is scheduled besides those at regular intervals if clinically necessary. The treatment decisions at each visit are made at discretion of treating rheumatologists based on disease activity heading for remission. The participants provided their informed consent to participate in this study.

All patients in the TARRA cohort fulfilled the 1987 ACR classification criteria ${ }^{22}$ or 2010 ACR/EULAR classification criteria for RA. ${ }^{23}$ The inclusion criteria were as follows: (1) visited the outpatient clinic between January 2009 and December 2019; (2) started GC and concomitant csDMARDs therapy at the initial visit; (3) had over 1-year follow-up with at least three visits. The exclusion criteria were as follows: (1) GC was used for comorbidities other than RA; (2) presenting another disease (eg, polymyalgia rheumatica, systemic lupus erythematosus) or certain condition (eg, pregnancy, lactation) that might influence GC tarping; (3) concomitant use of a bDMARD or targeted synthetic DMARDs (tsDMARD) at the initiation of GC and csDMARDs;
(4) GC initiation in other centres without sufficient previous clinical data.

\section{Clinical assessments and data collection}

The data of each eligible participant were collected from the timepoint of commencing GC treatment to December 2020. The collected data included (1) demographics: sex, age; (2) clinical features of RA: disease duration, titres of rheumatoid factor and anticyclic citrullinated peptides, tender joint counts and swollen joint counts, erythrocyte sedimentation rate (ESR), $\mathrm{C}$ reactive protein (CRP), patient's global assessment (PGA), and evaluator's global assessment (EGA); (3) treatment details at each visit (GC; methotrexate (MTX); leflunomide (LEF); hydroxychloroquine (HCQ); sulfasalazine (SSZ); tripterygium wilfordii Hook F (TII)); non-steroidal anti-inflammatory drugs (NSAIDs)).

In this study, a patient who never received DMARDs or was on DMARDs treatment for less than 3 months at the initiation of GC was defined as a DMARD-naive patient. The level of disease activity at each visit was measured by disease activity score based on 28-joint count and ESR (DAS28-ESR), ${ }^{24}$ simplified disease activity index (SDAI), ${ }^{25}$ and clinical disease activity index (CDAI). ${ }^{26}$ When analysing disease activity at the point of GC discontinuation, the data retroactively closest to the discontinuation day were used. The formula for calculating the composite disease activity scores with corresponding definitions of remission are as below:

$$
\begin{aligned}
& \text { 1. } \mathrm{DAS} 28-\mathrm{ESR}=(0.56 \sqrt{ }[\mathrm{TJC} 28]+0.28 \sqrt{ }[\mathrm{SJC} 28]+\ln [\mathrm{ESR}] \\
& +0.014 \times \mathrm{PGA})<2.6 \\
& \text { 2. } \mathrm{SDAI}=(\mathrm{TJC} 28+\mathrm{SJC} 28+\mathrm{CRP}+\mathrm{PGA}+\mathrm{EGA}) \leq 3.3 \\
& \text { 3. } \mathrm{CDAI}=(\mathrm{TJC} 28+\mathrm{SJC} 28+\mathrm{PGA}+\mathrm{EGA}) \leq 2.8
\end{aligned}
$$

\section{Discontinuation of GC}

The prescribed GC dosage was checked at each visit and converted to prednisolone (PSL) equivalent dose. The decision of tapering or discontinuing GC was made by treating rheumatologists. Discontinuation of GC in a patient at discretion of treating physician during follow-up was regarded as GC discontinuation. Adding of any $b / t s D M A R D$ for the purpose of facilitating GC tapering during follow-up was regarded as failure of GC discontinuation in the context of csDMARDs, and the data before b/tsDMARD initiation were analysed.

In this study, a patient who discontinued GC however with concomitant csDMARDs increment or new csDMARDs added after 3 months of GC initiation was defined as GC discontinuation with csDMARDs added or csDMARDs increment. csDMARD combination was defined as taking more than one of MTX, LEF, HCQ, SSZ or TII. The relapse profile within 6 months after GC withdrawal was also assessed. A patient was defined as flare in this study if one of the following criteria was satisfied within 6 months after GC withdrawal: (1) increase of DAS2 8 beyond $0.60^{27}$; (2) GC reinitiation; (3) adding or increment of any csDMARDs, b/tsDMARD; (4) rheumatologist's judgement.

\section{Statistical analysis}

Patient characteristics were presented as mean (SD) or median and IQR depending on the level of resemblance to the normal distribution. Absolute and relative frequencies were reported for categorical variables. Remission rates were measured by aforementioned definitions, reported as percentages with 95\% CIs. The trends of PSL dose, disease activity and remission rate during the study period were analysed using generalised estimating equations with an unstructured working correlation matrix and 


\begin{tabular}{|c|c|}
\hline & \\
\hline \multicolumn{2}{|l|}{ Basic characteristics } \\
\hline Age at GC initiation (years) & $55.9 \pm 14.5$ \\
\hline Female, \% (n) & $82.6 \%(171)$ \\
\hline Disease duration (months) & $24.0(8.5-96.0)$ \\
\hline DMARD naïve & $53.1 \%(110)$ \\
\hline RF positive, \% (n) & $73.2 \%(55 / 205)$ \\
\hline Anti-CCP positive, \% (n) & $79.5 \%(151 / 190)$ \\
\hline \multicolumn{2}{|l|}{ Baseline disease activity measures } \\
\hline TJC28 & $4(2-10)$ \\
\hline SJC28 & $3(1-5)$ \\
\hline $\mathrm{PGA}, 0-10 \mathrm{~cm}$ & $5(3-6)$ \\
\hline $\mathrm{EGA}, 0-10 \mathrm{~cm}$ & $4(2-6)$ \\
\hline ESR, mm/hour & $38(17-60)$ \\
\hline CRP, mg/dL & $1.30(0.47-3.74)$ \\
\hline DAS28-ESR & $4.76(3.69-5.96)$ \\
\hline SDAI & $18.7(10.5-32.4)$ \\
\hline CDAl & $16.0(9.0-29.0)$ \\
\hline \multicolumn{2}{|l|}{ Initial therapy paradigm } \\
\hline MTX, \% (n) & $75.4 \%(156)$ \\
\hline LEF, \% (n) & $53.1 \%(110)$ \\
\hline $\mathrm{HCQ}, \%(\mathrm{n})$ & $55.6 \%(115)$ \\
\hline $\mathrm{SSZ}, \%(\mathrm{n})$ & $9.2 \%(19)$ \\
\hline TII, \% (n) & $13.0 \%(27)$ \\
\hline NSAIDs, \% (n) & $13.0 \%(27)$ \\
\hline DMARD combination, \% (n) & $81.1 \%(163)$ \\
\hline PSL equivalent dose, mg/day & $10.0(5.0-10.0)$ \\
\hline \multicolumn{2}{|c|}{$\begin{array}{l}\text { Values are presented as mean (SD) or median (IQR), as applicable. } \\
\text { Anti-CCP, anti-cyclic citrullinated peptides; CDAl, clinical disease activity index; CRP, } \\
\text { C reactive protein; DAS28, disease activity score based on 28-joint count; DMARDs, } \\
\text { disease-modifying antirheumatic drugs; EGA, evaluator's global assessment; ESR, } \\
\text { erythrocyte sedimentation rate; HCQ, hydroxychloroquine; LEF, leflunomide; MTX, } \\
\text { methotrexate; PGA, patient's global assessment; PSL, prednisolone; RF, rheumatoid } \\
\text { factor; SDAI, simplified disease activity index; SJC, swollen joint count; SSZ, } \\
\text { sulfasalazine; TJC, tender joint count. }\end{array}$} \\
\hline
\end{tabular}

a robust estimation for the covariance matrix. The comparisons of clinical characteristics and treatment profiles for the patients who discontinued GC at the points of GC initiation and discontinuation were performed by the non-parametric Wilcoxon signed rank sum test for paired skewed continuous variables and the $\mathrm{McNemar} \chi^{2}$ test for paired categorical data. Comparisons between DMARD-naïve and DMARD-experienced patients were analysed by Student's t-tests for normally distributed continuous variables, Mann-Whitney U tests for skewed continuous variables, and $\chi^{2}$ tests for categorical variables. The cumulative probability of GC discontinuation and median time to GC discontinuation was calculated by the Kaplan-Meier method. All the analyses were done by SPSS V.20.0. Microsoft Excel 2010, GraphPad Prism V.8.0 and R software were used to produce the graphs. The level of significance was set at a two-sided $\mathrm{p}$ value less than 0.05 .

\section{RESULTS}

\section{Patient characteristics at the initiation of GC}

The enrolment flow chart is shown in online supplemental figure S1. In total, 207 consecutive patients contributing 2409 clinic visits were included with a low risk of selection bias (online supplemental table S1 and S2). The baseline characteristics of the 207 patients are presented in table 1 . At the start of GC,

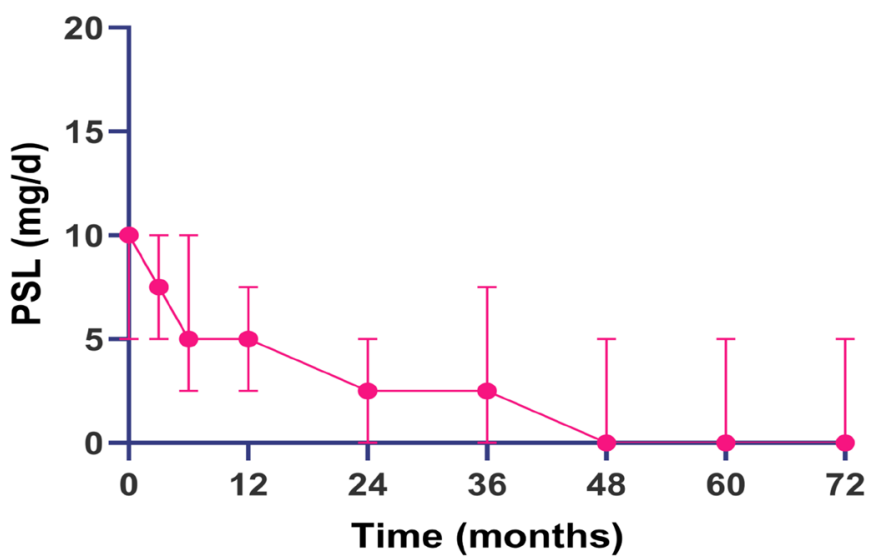

\begin{tabular}{|l|r|r|r|r|r|r|r|r|r|}
\hline Months & 0 & 3 & 6 & 12 & 24 & 36 & 48 & 60 & 72 \\
\hline No.pts & 207 & 196 & 191 & 202 & 147 & 103 & 77 & 49 & 38 \\
\hline
\end{tabular}

Figure 1 The changes in prednisone dose in patients with rheumatoid arthritis who started glucocorticoids with concomitant conventional synthetic disease modifying antirheumatic drugs during the follow-up period. Values are presented as median (IQRs). PSL, prednisolone.

the mean age (SD) of study participants was 55.9 (14.5) years with median (IQR) disease duration of 24 (8.5-96.0) months. Most patients were female and serological positive. Over half of participants (110/207) were DMARD naïve. At the initiation of GC, the majority of patients had moderate-to-high disease activity. The median (IQR) follow-up duration was 38.6 (20.760.3) months.

Therapeutically, the top three initiating PSL daily doses were $10 \mathrm{mg}$ (100 patients), $5 \mathrm{mg}$ (51 patients) and $15 \mathrm{mg}$ (14 patients), with the median (IQR) dose of $10.0(5.0-10.0) \mathrm{mg}$. MTX (75.4\%), HCQ (55.6\%) and LEF (53.1\%) were most frequently prescribed concomitant csDMARDs. At baseline, csDMARD combination was applied in $81.1 \%$ of patients, and the first two double combination regimens were MTX plus HCQ and MTX plus LEF. Besides, 27 (13.0\%) patients also received concomitant NSAIDs at the start of GC.

Changes in PSL dose and disease activity in the entire cohort Of the 207 patients, data on PSL dose (disease activity) at baseline, months $3,6,12,24,36,48,60$ and 72 were available in 207 (207), 196 (189), 191 (185), 202 (195), 147 (148), 103 (104), 77 (71), 49 (47) and 39 (32) patients. The median time interval between the timepoint of disease activity assessment and GC discontinuation was 0 day (IQR: 0-14).

Overall, the median PSL dose was significantly decreased during the observation period ( $\mathrm{p}<0.001$; figure 1$)$. The median PSL daily dose of $10(5-10) \mathrm{mg}$ at initiation was reduced to $7.5(5-10) \mathrm{mg}$ at 3 months and $5.0(2.5-10) \mathrm{mg}$ at 6 months. In the subsequent follow-up period, the median dose was more slowly reduced to $5(2.5-7.5) \mathrm{mg}$ at 12 months, $2.5(0-7.5) \mathrm{mg}$ at 24 months, $2.5(0-5.0) \mathrm{mg}$ by 36 months and finally to $0 \quad(0-5.0) \mathrm{mg}$ by 48 months (maintained to 72 months). Regarding the disease control, significant improvements were also witnessed in disease activity and remission/LDA rates, measured by DAS28-ESR, SDAI and CDAI during the first 6 months $(p<0.001$; figure 2$)$. In the subsequent follow-up period, disease activity and remission/LDA rate were relatively stable with marginal fluctuation. 
A

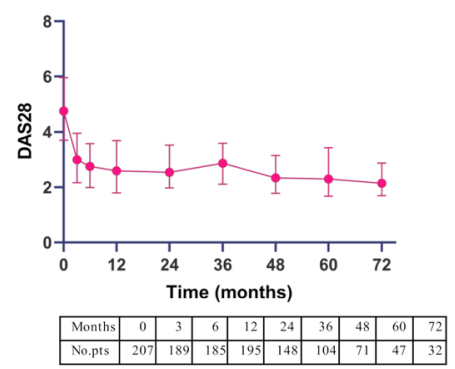

D

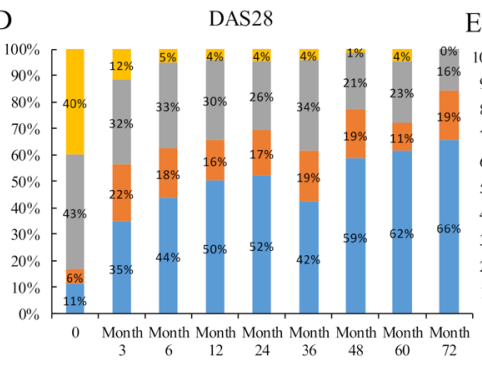

RFM = IDA -MDA

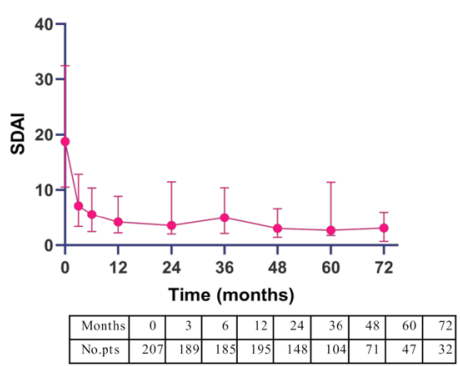

E
C

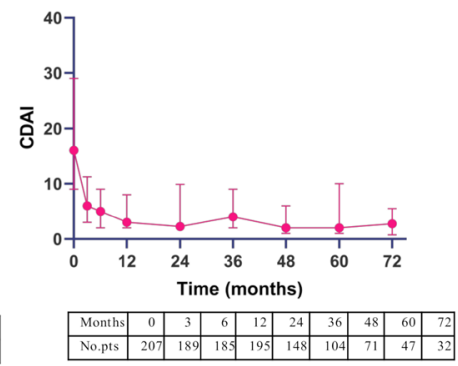

F

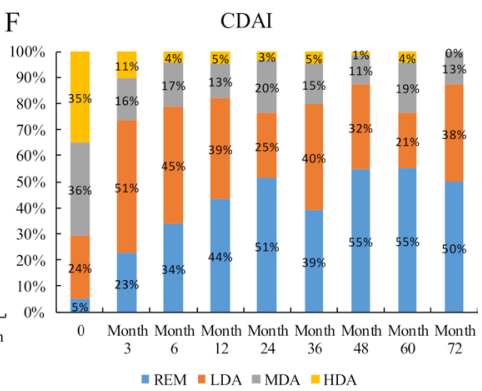

Figure 2 The changes in disease activity in patients with rheumatoid arthritis who started glucocorticoids with concomitant conventional synthetic disease-modifying antirheumatic drugs, measured by (A) DAS28, (B) SDAI, (C) CDAl, and the changes in the percentage of remission, as well as low, moderate and high disease activity measured by (D) DAS28, (E) SDAI, (F) CDAl, during the follow-up period. Values are presented as median (IQRs). CDAl, clinical disease activity index; csDMARDs, conventional synthetic disease-modifying antirheumatic drugs; DAS28, disease activity score based on 28-joint count; SDAl, simplified disease activity index.

\section{The frequency of GC discontinuation in the entire cohort}

During a median (IQR) follow-up of 38.6 (20.7-60.3) months, 124 out of 207 (59.9\%) patients finally discontinued GC at the discretion of treating rheumatologists. Kaplan-Meier estimate of the probability of GC discontinuation after initiating GC with concomitant csDMARDs in our patients is presented in figure 3. The cumulative probability of GC discontinuation was $9.7 \%$ at 6 months, $26.6 \%$ at year $1,48.0 \%$ at year 2, 58.6\% at year 3, and the calculated median time (IQR) to GC discontinuation was 27.0 (19.2-34.8) months. Among the 124 patients who

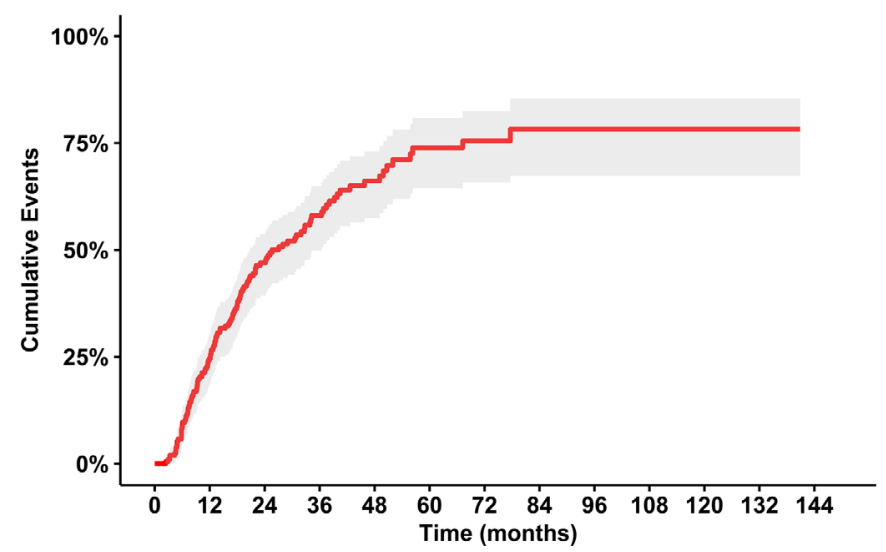

Number at risk

\begin{tabular}{|ccccccccccccc}
207 & 156 & 86 & 51 & 28 & 19 & 11 & 7 & 6 & 3 & 2 & 1 & 0 \\
\hline 0 & 12 & 24 & 36 & 48 & $\begin{array}{l}1 \\
1\end{array}$ & 72 & 84 & 96 & 108 & 120 & 132 & 144 \\
Time (months)
\end{tabular}

Figure 3 Kaplan-Meier curve with cumulative probability of glucocorticoids discontinuation in patients with rheumatoid arthritis who started glucocorticoids with concomitant csDMARDs during the follow-up period. csDMARDs, conventional synthetic disease-modifying antirheumatic drugs. discontinued GC, the median time (IQR) to GC withdrawal was 13.6 (8.0-24.6) months. Additionally, the rates of GC discontinuation stratified by follow-up duration showed a general ascending trend as observation period increases, however statistically insignificant (online supplemental table S3). We also compared the frequency of GC discontinuation and time to GC discontinuation between the patients at initial visit before and after 2014, showing significantly higher and faster GC discontinuation over time (before vs after 2014: $56.5 \%$ vs $64.1 \% ; 36.0$ vs 20.0 months; online supplemental table S4 and figure S2).

\section{The GC discontinuation in DMARD-naïve and DMARD- experienced patients}

Of the 207 patients, 110 were DMARD-naïve and 97 were DMARD-experienced. Their demographics and baseline clinical characteristics were roughly comparable (online supplemental table S5). During follow-up, 41 and 42 patients discontinued GC in DMARD-naïve and DMARD-experienced groups. In DMARD-naïve patients, the cumulative probabilities of GC discontinuation were $12.7 \%, 30.0 \%, 50.9 \%$ and $60.6 \%$ at month 6 , years 1,2 , and 3 , with calculated median time to GC cessation of 24 months (online supplemental figure S3). In DMARDexperienced patients, the corresponding cumulative probabilities of GC discontinuation were $6.2 \%, 22.7 \%, 44.7 \%, 56.5 \%$, with calculated median time to GC cessation of 32 months. No statistical difference was detected between the two groups (long-rank: $\mathrm{p}=0.621$ ), but seemingly faster GC discontinuation in DMARDnaïve patients (online supplemental figure S3). Additionally, we compared the GC discontinuation in DMARD-naïve patients with initial visit before 2014 and after 2014, similarly showing significantly higher and quicker GC discontinuation after 2014 (before vs after 2014: $58.6 \%$ vs $67.3 \%$; 38.0 vs 18.0 months; online supplemental table S4 and figure S4).

Moreover, we analysed the GC discontinuation between 77 early patients (disease duration $\leq 1$ year) and 33 established 
A

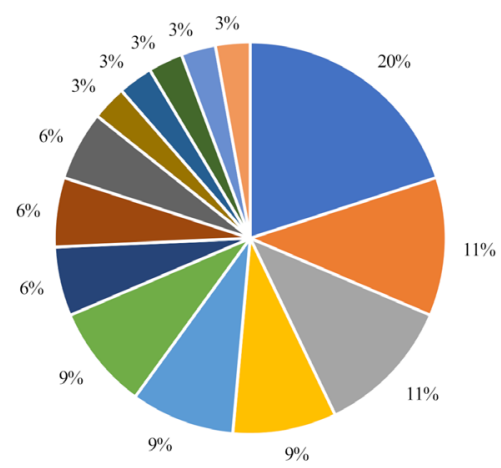

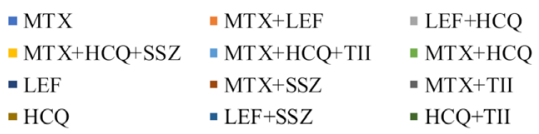

$\mathrm{B}$

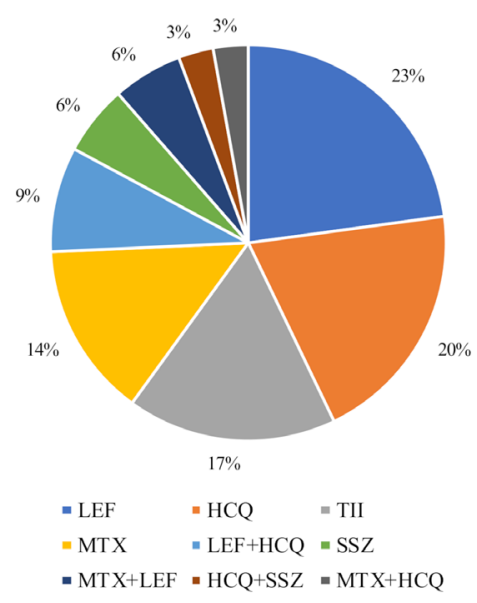

$\mathrm{C}$

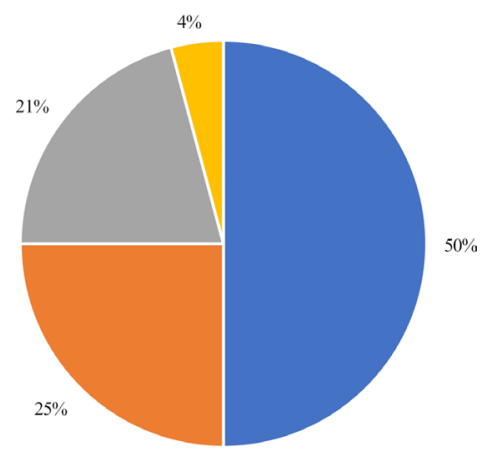

- $\mathrm{GC}$ re-initiation

- increasing DAS28 beyond 0.60

more than 1

" DMARDs added or csDMARs increment

$=\mathrm{MTX}+\mathrm{LEF}+\mathrm{HCQ} \quad=\mathrm{MTX}+\mathrm{LEF}+\mathrm{SSZ}$

Figure 4 (A) The details of prescribed regimen before conventional synthetic disease-modifying antirheumatic drugs (csDMARDs) added or csDMARDs increment in 35 patients with rheumatoid arthritis (RA) who discontinued glucocorticoids with csDMARDs added or increment. (B) The details of csDMARDs added or increment in 35 patients with RA who discontinued glucocorticoids. (C) The reasons judged as flare within 6 months after glucocorticoids withdrawal in 24 patients with RA. MTX, methotrexate; LEF, leflunomide; HCQ, hydroxychloroquine; SSZ, sulfasalazine; TII, tripterygium wilfordii Hook F.

patients in 110 DMARD-naïve patients. In early groups, the cumulative probabilities of GC discontinuation were $11.7 \%$, $29.9 \%, 53.3 \%$ and $63.5 \%$ at month 6 , year 1 , year 2 , and year 3 , with calculated median time to GC cessation of 22 months. In the established groups, the corresponding cumulative probabilities of GC discontinuation were 15.2\%, 30.3\%, 44.6\% and $53.1 \%$, with calculated median time to GC cessation of 34 months (online supplemental figure S5).

\section{The characteristics of patients who discontinued GC}

Of the 124 patients who stopped GC, 28.2\% (35/124) experienced institution of new csDMARDs or concomitant csDMARDs increment after 3 months of GC start. The detailed information on the prescribed regimens before csDMARDs added or csDMARDs increment is provided in figure 4A. The commonly added or increased csDMARDs during GC tapering were, in order, LEF (23\%), HCQ (20\%), TII (17\%) and MTX (14\%). The detailed information is provided in figure $4 \mathrm{~B}$.

For the 124 patients who discontinued GC, their median DAS28 was dramatically declined from 4.76 (3.67-5.95) at the point of GC initiation to $2.51(1.69-3.39)$ at the point of GC withdrawal $(\mathrm{p}<0.001)$. The same trend was also observed in SDAI (from 18.2 to 3.1), CDAI (from 16.0 to 3.0), and each individual component $(\mathrm{p}<0.0001$; table 2$)$. Approximately half of 124 patients reached remission at the point of GC discontinuation. The detailed changes in the proportions of achieving remission and LDA are presented in online supplemental figure S6. In terms of treatment paradigm, significant increase in HCQ usage $(p<0.0001)$ and decrease in NSAIDs use $(p=0.002)$ were observed at GC discontinuation compared with those at GC initiation (table 2). There was no significant finding for the remaining therapeutic patterns.

We further analysed the relapse profiles within 6 months after GC discontinuation. Among the 124 patients who stopped GC, relevant clinical data were available in 115 patients. Flare free within 6 months after GC withdrawal was observed in 91 (79.1\%) patients. The most common reasons judged for flare
Table 2 The characteristics of 124 patients with RA who discontinued glucocorticoids at the points of glucocorticoids initiation and discontinuation

\begin{tabular}{|c|c|c|c|}
\hline Characteristics & GC initiation visit & $\begin{array}{l}\text { GC discontinuation } \\
\text { visit }\end{array}$ & $P$ value \\
\hline \multicolumn{4}{|c|}{ Disease activity measures } \\
\hline TJC28 & $4(1-9)$ & $0(0-2)$ & $<0.0001$ \\
\hline SJC28 & $3(1-6)$ & $0(0-1)$ & $<0.0001$ \\
\hline PGA, $0-10 \mathrm{~cm}$ & $5(3-6)$ & $1(1-3)$ & $<0.0001$ \\
\hline $\mathrm{EGA}, 0-10 \mathrm{~cm}$ & $5(3-6)$ & $1(0.5-2)$ & $<0.0001$ \\
\hline ESR, mm/hour & $37(17-62)$ & $18(8-29)$ & $<0.0001$ \\
\hline CRP, mg/dL & $1.28(0.46-4.11)$ & $0.33(0.20-0.77)$ & $<0.0001$ \\
\hline DAS28-ESR & $4.76(3.67-5.95)$ & $2.51(1.69-3.39)$ & $<0.0001$ \\
\hline SDAI & $18.2(10.8-31.8)$ & $3.1(1.8-9.0)$ & $<0.0001$ \\
\hline CDAl & $16.0(9.0-27.0)$ & $3.0(1.5-8.0)$ & $<0.0001$ \\
\hline \multicolumn{4}{|c|}{ Treatment paradigm, \% (n) } \\
\hline MTX & $78.2 \%(97)$ & $78.2 \%(97)$ & 1.00 \\
\hline LEF & $50.8 \%(63)$ & $50.0 \%(62)$ & 1.00 \\
\hline $\mathrm{HCQ}$ & $51.6 \%(64)$ & $61.3 \%(76)$ & $<0.0001$ \\
\hline SSZ & $10.5 \%(13)$ & $11.3 \%(14)$ & 1.00 \\
\hline TII & $12.1 \%(15)$ & $11.3 \%(14)$ & 1.00 \\
\hline NSAIDs & $8.9 \%(11)$ & $2.4 \%(1)$ & 0.002 \\
\hline $\begin{array}{l}\text { DMARDs } \\
\text { combination }\end{array}$ & $81.1 \%(99)$ & $81.1 \%(99)$ & 0.690 \\
\hline $\begin{array}{l}\text { Double } \\
\text { combination }\end{array}$ & $55.6 \%(69)$ & $46.0 \%(57)$ & 0.570 \\
\hline $\begin{array}{l}\text { Triple } \\
\text { combination }\end{array}$ & $24.2 \%(30)$ & $31.5 \%(39)$ & 0.495 \\
\hline
\end{tabular}

CDAl, clinical disease activity index; CRP, C reactive protein; DAS28, disease activity score based on 28-joint assessment; DMARDs, disease-modifying antirheumatic drugs; EGA, evaluator's global assessment; ESR, erythrocyte sedimentation rate; HCQ, hydroxychloroquine; LEF, leflunomide; MTX, methotrexate; NSAIDs, non-steroidal anti-inflammatory drugs; PGA, patient's global assessment; SDAI, simplified disease activity index; SJC, swollen joint count; SSZ, sulfasalazine; TII, tripterygium wilfordii Hook F; TJC, tender joint count. 
was GC reinitiation $(50 \%, 12 / 24)$ and increase of DAS28 over $0.60(25 \%, 6 / 24$; figure 4C).

\section{DISCUSSION}

GC has been proven to improve disease activity, physical function and structural outcomes in $\mathrm{RA},{ }^{1-5}$ and recommended as bridging therapy for csDMARDs to exhibit their efficacy in current guidelines. ${ }^{278}$ Because of safety concerns on longterm exposure, even at low-dose, GC should be tapered and ultimately stopped, as rapidly as clinically feasible. In EULAR recommendations, the therapy is considered as failure if GC cannot be withdrawn within acceptable time frame, and adding a b/tsDMARD should be considered. ${ }^{27}$ Previous clinical investigations have evaluated the feasibility of GC discontinuation by introducing the bDMARD or tofacitinib. ${ }^{27-32}$ For example, Inoue et al analysed 80 patients with RA taking a median PSL dose of $5.0(1.0-10.0) \mathrm{mg} /$ day at bDMARDs initiation. ${ }^{31}$ Over a median follow-up of 33.1 months, $31.3 \%$ of participants discontinued GC. Recently, a pooled analysis of two multicentre, open-label studies with 1434 patients with RA receiving GC (mean dose of PSL $6.1 \mathrm{mg} /$ day) and tofacitinib $5 \mathrm{mg}$ or $10 \mathrm{mg}$ two times a day showed only $22.2 \%$ patients discontinued GC by year $3 .{ }^{32} \mathrm{~A}$ very recent randomised controlled trial investigated continuing and tapering GC in patients with RA in LDA/remission with background therapy of tocilizumab. ${ }^{27}$ Patients continuing PSL at $5 \mathrm{mg} /$ day achieved safe and better disease control than tapering group, although only two-thirds of them were able to taper GC. So far, the trajectory and characteristics of GC cessation with concomitant csDMARDs before eliciting a b/tsDMARD remain completely unknown.

The present study first provided real-world evidence on the GC tapering and discontinuation in patients with RA commencing GC and concomitant csDMARDs therapy. Overall, the median PSL dose was reduced by $50 \%$ in the first 6 months, then more slowly in subsequent period and finally to zero by 48 months. The cumulative probabilities of GC discontinuation were $26.6 \%, 48.0 \%$ and $58.6 \%$ at years 1,2 and 3 . The calculated median time to GC discontinuation of 27 months was dramatically longer than the time frame recommended by EULAR (3-6 months in 2016 and 3 months in 2019). ${ }^{27} \mathrm{~A}$ few reasons for the phenomena. First, GC has been pretty commonly prescribed in practice for decades, but few data to guide clinicians to taper GC in RA. ${ }^{14}{ }^{15}$ Second, despite of tapering and discontinuing GC in RA has been proposed for years, only recently with the availability of more targeted DMARDs, more attention is paid to the cumulative evidence of long-term GC associated side effects. As shown in our cohort of patients with initial visit after 2014, compared with those before 2014, the frequency of GC discontinuation was significantly increased $(64.1 \%$ vs $56.5 \%)$ with significantly shortened time to GC discontinuation (20.0 vs 36.0 months). These reflect the evolution of concepts of GC use in RA over time. Third, EULAR recommends to add a b/tsDMARD when GC cannot be discontinued within 3 months in patients receiving GC cotherapy with csDMARDs. Largely limited by enormous economic burden as well as high prevalence of tuberculosis and hepatitis in China, the prescription of b/tsDMARD was extremely low, from $4.0 \%$ to $9.1 \% .^{20} 33-35$ The low accessibility to b/tsDMARD might be attributable to the longer time to GC discontinuation in the context of csDMARDs therapies in daily practice. The real-life long-term GC use, on the other hand, makes our study of complete dynamical trajectory of GC tapering and discontinuation in the context of csDMARDs therapy possible. Although there is a gap between real-world practice and current guidelines in GC discontinuation, we are delighted to see the gap is becoming narrow, with a significantly higher and faster GC discontinuation in more recent years. These results not only reflect the evolution of concepts of GC use in RA over time, but also confirm the feasibility to shorten the time to GC discontinuation, even within the recommended timeframe in guidelines, especially with better accessibility of targeted DMARDs. An evidence-based protocol for GC tapering and discontinuation is indeed required to guide clinicians to taper GC for patients with RA.

There are several advantages in the present study. First, all enrolled patients with RA received their first dose GC and concomitant csDMARDs at the initial visit. In this way, the actual and dynamical trajectory of GC and characteristic of GC discontinuation can be completely depicted. Second, the current study provides important information regarding the risk of RA relapse after GC withdrawal. This was scarcely discussed in previous reports. Strengths of this study also include the setting of real world.

We acknowledge several limitations in this study. First, the findings of a single-centre retrospective study may limit the external generalisability, though internal generalisability is greatest. Second, the sample size was relatively small. Nevertheless, no study elucidating the dynamical trajectory and characteristics of GC discontinuation in patients with RA who start GC with concomitant csDMARDs was ever published before. The current study indeed adds important information to our understanding of GC discontinuation in RA. Last, the adverse events are not discussed. In the study, we focused on the dynamical trajectory of GC tapering and corresponding disease activity in patients with RA who started GC with concomitant csDMARDs.

\section{CONCLUSIONS}

GC is feasibly discontinued in real-life setting when disease activity is favourable, mostly without short-term flare, in patients who newly start GC with concomitant csDMARDs. But the withdrawal time is far from reaching the recommended time frame, suggesting the gap between real-world practice and current guidelines.

Acknowledgements The authors would like to thank all the patients and rheumatology nurses who contributed to our study. The authors also acknowledge the assistance of Dr Shiyu Xiao for his assistance in generating figure 3.

Contributors ZZ conceived of the study, participated in its design and coordination, and critically revised the manuscript. WX had full access to all of the data collection, analysis, interpretation and drafted the manuscript. HH, GL, Y-JH, YG, $Y W, X-r D, J Z, Y G, L J, X Z$ and $Z S$ contributed to the process of data collection. All the authors listed have approved the enclosed manuscript.

Funding This work was supported by the National Natural Science Foundation of China (grant number: 81771740, 81801611, 81971524).

Competing interests None declared.

Patient consent for publication Not required.

Ethics approval This study was approved by the Ethics Committee of Peking University First Hospital. The TARRA cohort was approved by the Institutional Review Board (IRB) of our hospital (IRB number 2014-785).

Provenance and peer review Not commissioned; externally peer reviewed.

Data availability statement All data relevant to the study are included in the article or uploaded as supplementary information. All data relevant to the study are included in the article or uploaded as supplementary information.

Supplemental material This content has been supplied by the author(s). It has not been vetted by BMJ Publishing Group Limited (BMJ) and may not have been peer-reviewed. Any opinions or recommendations discussed are solely those of the author(s) and are not endorsed by BMJ. BMJ disclaims all liability and responsibility arising from any reliance placed on the content. Where the content includes any translated material, BMJ does not warrant the accuracy and reliability of the translations (including but not limited to local regulations, clinical guidelines, 
terminology, drug names and drug dosages), and is not responsible for any error and/or omissions arising from translation and adaptation or otherwise.

\section{ORCID iDs}

Wenhui Xie http://orcid.org/0000-0002-3881-0266

Hong Huang http://orcid.org/0000-0002-1468-1586

Juan Zhao http://orcid.org/0000-0002-9547-887X

Zhuoli Zhang http://orcid.org/0000-0001-7219-9141

\section{REFERENCES}

1 Conn DL. The story behind the use of glucocorticoids in the treatment of rheumatoid arthritis. Semin Arthritis Rheum 2021;51:15-19.

2 Smolen IS, Landewé RBM, Bijlsma JWJ, et al. EULAR recommendations for the management of rheumatoid arthritis with synthetic and biological disease-modifying antirheumatic drugs: 2019 update. Ann Rheum Dis 2020;79:685-99.

3 Akdemir G, Heimans L, Bergstra SA, et al. Clinical and radiological outcomes of 5-year drug-free remission-steered treatment in patients with early arthritis: improved study. Ann Rheum Dis 2018;77:111-8.

4 Stouten V, Westhovens R, Pazmino S, et al. Effectiveness of different combinations of DMARDs and glucocorticoid bridging in early rheumatoid arthritis: two-year results of CareRA. Rheumatology 2019;58:2284-94.

5 Hetland ML, Haavardsholm EA, Rudin A, et al. Active conventional treatment and three different biological treatments in early rheumatoid arthritis: phase IV investigator initiated, randomised, observer blinded clinical trial. BMJ 2020;371:m4328.

6 Santiago T, Voshaar M, de Wit M, et al. Patients' and rheumatologists' perspectives on the efficacy and safety of low-dose glucocorticoids in rheumatoid arthritis-an international survey within the GLORIA study. Rheumatology 2021;4:keaa785.

7 Smolen JS, Landewé R, BijJsma J, et al. EULAR recommendations for the management of rheumatoid arthritis with synthetic and biological disease-modifying antirheumatic drugs: 2016 update. Ann Rheum Dis 2017;76:960-77.

8 Singh JA, Saag KG, Bridges SL, et al. 2015 American College of rheumatology guideline for the treatment of rheumatoid arthritis. Arthritis Rheumatol 2016;68:1-26.

9 Roubille C, Rincheval N, Dougados M, et al. Seven-Year tolerability profile of glucocorticoids use in early rheumatoid arthritis: data from the ESPOIR cohort. Ann Rheum Dis 2017;76:1797-802.

10 Roubille C, Coffy A, Rincheval N, et al. Ten-Year analysis of the risk of severe outcomes related to low-dose glucocorticoids in early rheumatoid arthritis. Rheumatology 2020;15:keaa850.

11 Xie W, Yang X, Ji L, et al. Incident diabetes associated with hydroxychloroquine, methotrexate, biologics and glucocorticoids in rheumatoid arthritis: a systematic review and meta-analysis. Semin Arthritis Rheum 2020;50:598-607.

12 George MD, Baker JF, Winthrop K, et al. Risk for Serious Infection With Low-Dose Glucocorticoids in Patients With Rheumatoid Arthritis : A Cohort Study. Ann Intern Med 2020;173:870-8.

13 Abtahi S, Driessen JHM, Burden AM, et al. Concomitant use of oral glucocorticoids and proton pump inhibitors and risk of osteoporotic fractures among patients with rheumatoid arthritis: a population-based cohort study. Ann Rheum Dis 2021;80:423-31.

14 Wallace BI, Wallace DM, Waljee AK, et al. Evidence to support or guide glucocorticoid tapering in rheumatoid arthritis is lacking. Ann Rheum Dis 2019;78:1733-4.

15 Volkmann ER, Rezai S, Tarp S, et al. We still don't know how to taper glucocorticoids in rheumatoid arthritis, and we can do better. J Rheumatol 2013:40:1646-9.

16 Xie W, Li G, Huang H, et al. How to define Boolean low disease activity in rheumatoid arthritis: experience from a large real-world cohort. Rheumatol Ther 2021;8:289-301.
17 Ji L, Xie W, Li G, et al. Maintenance to target was associated with radiological outcomes in patients with rheumatoid arthritis: a real-world observational cohort study. Clin Rheumatol 2021;40:1307-15.

18 Xie W, Li J, Zhang Z. The impact of different criteria sets on early remission and identifying its predictors in rheumatoid arthritis: results from an observational cohort (2009-2018). Clin Rheumatol 2020;39:381-9.

19 Xie W, Li J, Zhang X, et al. Sustained clinical remission of rheumatoid arthritis and its predictive factors in an unselected adult Chinese population from 2009 to 2018. Int J Rheum Dis 2019;22:1670-8.

20 Xie W, Li J, Zhang X, et al. Trends in the activity of rheumatoid arthritis as the consequence of treat-to-target strategy: eight-year data from 2009 to 2016. Clin Exp Rheumatol 2018:36:820-8.

21 Zhang H, Zhang H, Gao D, et al. Overlapping Sjogren's syndrome reduces the probability of reaching target in rheumatoid arthritis patients: a propensity score matched real-world cohort from 2009 to 2019. Arthritis Res Ther 2020;22:100.

22 Arnett FC, Edworthy SM, Bloch DA, et al. The American rheumatism association 1987 revised criteria for the classification of rheumatoid arthritis. Arthritis Rheum 1988;31:315-24

23 Aletaha D, Neogi T, Silman AJ, et al. 2010 rheumatoid arthritis classification criteria: an American College of Rheumatology/European League against rheumatism collaborative initiative. Ann Rheum Dis 2010;69:1580-8.

24 Fransen J, Creemers MCW, Van Riel PLCM. Remission in rheumatoid arthritis: agreement of the disease activity score (DAS28) with the Ara preliminary remission criteria. Rheumatology 2004:43:1252-5.

25 Smolen JS, Breedveld FC, Schiff MH, et al. A simplified disease activity index for rheumatoid arthritis for use in clinical practice. Rheumatology 2003;42:244-57.

26 Aletaha D, Nell VPK, Stamm T, et al. Acute phase reactants add little to composite disease activity indices for rheumatoid arthritis: validation of a clinical activity score. Arthritis Res Ther 2005;7:R796-806.

27 Burmester GR, Buttgereit F, Bernasconi C, et al. Continuing versus tapering glucocorticoids after achievement of low disease activity or remission in rheumatoid arthritis (SEMIRA): a double-blind, multicentre, randomised controlled trial. Lancet 2020:396:267-76.

28 Hirata S, Kohno H, Watanabe $\mathrm{H}$, et al. Tapering and discontinuation of oral glucocorticoids without deterioration of disease status in patients with rheumatoid arthritis under a stable treatment. Mod Rheumatol 2021;79:1-10.

29 Alten R, Nüßlein H, Galeazzi M, et al. Decreased use of glucocorticoids in biologicalexperienced patients with rheumatoid arthritis who initiated intravenous abatacept: results from the 2-year ACTION study. RMD Open 2016;2:e00228.

30 Shimizu Y, Tanaka E, Inoue E, et al. Reduction of methotrexate and glucocorticoids use after the introduction of biological disease-modifying anti-rheumatic drugs in patients with rheumatoid arthritis in daily practice based on the IORRA cohort. Mod Rheumatol 2018;28:461-7.

31 Inoue $\mathrm{M}$, Kanda $\mathrm{H}$, Tateishi $\mathrm{S}$, et al. Factors associated with discontinuation of glucocorticoids after starting biological disease-modifying antirheumatic drugs in rheumatoid arthritis patients. Mod Rheumatol 2020;30:58-63.

32 Fleischmann R, Wollenhaupt J, Cohen S, et al. Effect of discontinuation or initiation of methotrexate or glucocorticoids on tofacitinib efficacy in patients with rheumatoid arthritis: a post hoc analysis. Rheumatol Ther 2018;5:203-14.

33 Yu C, Li M, Duan X, et al. Chinese registry of rheumatoid arthritis (CREDIT): I. Introduction and prevalence of remission in Chinese patients with rheumatoid arthritis. Clin Exp Rheumatol 2018;36:836-40.

34 Wang G-Y, Zhang S-L, Wang X-R, et al. Remission of rheumatoid arthritis and potential determinants: a national multi-center cross-sectional survey. Clin Rheumatol 2015:34:221-30.

35 Zhu H, Li R, Da Z, et al. Remission assessment of rheumatoid arthritis in daily practice in China: a cross-sectional observational study. Clin Rheumatol 2018:37:597-605. 